\title{
The Application of ADS-B Technology in Aviation Management in China
}

\author{
Chen Wenhui, Chen Quanshan, Wang Yu, Chen Huan, He Yu, Ling Ze \\ Beijing Institute of Technology, Zhuhai, Guangdong Zhuhai, China, 519088
}

Keywords: ADS-B technology; China; aviation management; application

\begin{abstract}
In recent years, with the rapid development of China's social economy and the continuous improvement of people's income level, most people use airplanes, which are faster-moving travel tools, and the number of relative aircraft is increasing. The operational safety of aircraft will directly affect people's lives. Using ADS-B technology can effectively improve the accuracy of monitoring data and improve the quality of aviation management. This paper briefly analyzes the ADS-B technology, studies the development status of ADS-B technology in China, and explores the problems existing in the application of ADS-B technology and the development prospects of ADS-B system.
\end{abstract}

\section{Introduction}

With the development of China's social economy, China's transportation industry is also developing. As the best means of transportation for long-distance travel, aircraft has developed rapidly in recent years. At this stage, the number of aircraft in China continues to increase, and the number of flights is also increasing. It is precisely because of the above reasons that China's airborne area is relatively busy. If it is not strictly controlled and planned in the airborne area, it may cause a safety accident, and it is necessary to strengthen aviation management to ensure people's travel safety. Applying ADS-B technology to China's aviation management work can improve the quality of aviation management, which can reduce the probability of safety accidents and ensure people's travel safety.

\section{Overview of ADS-B Technology}

\subsection{ADS-B technology}

Broadcast-type automatic correlation monitoring belongs to a new type of aircraft operation monitoring technology. Its main purpose is to strengthen air traffic management and ensure the safety of travelers. In the future, ADS-B technology will replace the original radar monitoring technology for aircraft operation monitoring, so as to clarify the safety responsibility during flight and taxiing. Compared with traditional monitoring technology, the monitoring data collected by ADS-B technology has higher precision and wider coverage.

ADS-B technology utilizes a global satellite navigation system to receive aircraft position information in a timely and accurate manner, and transmits its information to aircraft or ground 
station users equipped with ADS-B facilities through data link system broadcasting, so as to ensure that the aircraft can remain safe. distance. After using advanced monitoring technology in the aircraft, the air traffic safety responsibility will be transferred from the traffic controller to the pilot, who can use their own information to maintain a safe distance from other aircraft. ADS-B technology mainly uses satellite receivers to receive corresponding satellite signals, and uses signal transmitters to transmit aircraft position information to other aircraft or ground station users. This advanced monitoring system can collect aircraft position information in real time, set the time label to this information and convert it into displacement digital code, and synthesize the aircraft type, flight number, speed and other data information, and broadcast the data information at regular intervals.

The ADS-B system mainly has the following application methods: First, the air-to-air application. The aircraft cockpit traffic information display can present aircraft traffic information in real time. The pilot can observe the operation of the aircraft around the air and maintain a safe distance from other aircraft, so that the pilot can actively prevent flight conflicts and improve flight safety. Secondly, Air-to-ground application. The ADS-B system can transmit the aircraft position information to the ground station users in real time. The ground control traffic management personnel can understand the operation of the aircraft according to the data information they have mastered, and carry out scientific control and command. Aircraft and vehicles operating at the airport also need to make full use of ADS-B technology to ensure a safe distance between each other. The application of the ADS-B monitoring system not only improves the utilization of the airspace system, but also improves the monitoring capability of the airport.

In short, ADS-B technology combines conflict monitoring, conflict avoidance, and air traffic control surveillance, which is important for improving the quality of air traffic management and promoting the stable development of the air traffic industry.

\subsection{ADS-B technical principle}

ADS-B technology will collect four-dimensional position information, other additional information, aircraft identification information, and category information of the aircraft. In addition, ADS-B technology can also collect data such as heading, airspeed, and customs. The ADS-B system uses the global satellite navigation system, inertial navigation system, inertial reference system and other systems to collect these data information.

The ADS-B system consists of the following components: First, the airborne part. This part can collect relevant data information such as aircraft position, flight speed, air pressure and other realtime information through global satellite navigation system, atmospheric data system and other systems, and broadcast the information such as data information and aircraft identification. This will allow other aircraft using the ADS-B system to understand this information with the ground users; Second, the ground segment. After the above information is obtained, the ground users can transmit and save the information to the air traffic control system and control and monitor it by the management personnel; the aircraft position information can be displayed on other aircraft using the ADS-B system after being processed. On the CDTI.

One of the most important human-machine interfaces in the ADS-B system is the CDTI, which displays air traffic management information such as navigation information, terrain warning information, and weather radar information to the pilot. Vehicles in the airport also need to install the ADS-B system, so airport scene managers can manage the airport scene vehicles to avoid illegally occupying the runway. 


\section{Development status of ADS-B technology in China}

Compared with developed countries, it is not too late for China's aviation industry to apply ADSB technology. After the founding of New China, the party and the government have always attached great importance to the development of aviation. With the rapid development of economy and science and technology, China has not promoted the stable and healthy development of the air transport industry in the western region, and began to strengthen research on the navigation system, and based on international Guided by the aviation organization's new navigation system development plan, the first new navigation system route based on ADS technology (also known as the L888 route) was established. In this route, the main application monitoring technology is ADS$\mathrm{C}$ technology, and a network management data center is set up in Beijing to complete air traffic management work. In 2000, the new system developed by China was officially promoted and applied in China after being tested and evaluated. In 2004, China, Beijing, Shanghai, and Guangzhou respectively built regional control centers, and installed an air traffic control automation system, which has ADS track processing functions. The study found that the newly developed and put into use new system can fully process and display the automatic related surveillance track related to ADS technology, and implement the "air traffic controller/pilot data link communication", which shows that China's aviation air traffic control equipment has ADS. Monitoring capabilities. The survey found that the number of airlines in China is increasing, and the number and number of aircraft are increasing. Some aircraft have installed new navigation systems and have two-way data capacity.

Although China's air transport industry has conducted in-depth research on navigation systems and air traffic surveillance technologies to meet the needs of social development, these studies have achieved certain results, they have not broken through the constraints of the original surveillance technology. Therefore, it is not very effective in solving the problems faced by air traffic management work and improving aviation safety. In recent years, while the rapid development of science and technology, ADS-B technology has gradually improved, which plays an important role in solving air traffic management problems and improving aviation safety. At this stage, ADS-B technology has been applied in civil aviation enterprises in China. The civil aviation unit that used this technology in China is the Civil Aviation Flight Academy of China. In 2009, the domestic ADS-B system was tested on the 7910 aircraft of China Aviation Flight Academy. It can be seen that China's ADS-B technology is gradually optimized and improved, and will be promoted and used in China in the future. The "Domestic ADS-B System" project is under the responsibility of the Dean of the Civil Aviation Flight Academy, Zheng Xiaoxuan, and is jointly researched by the Civil Aviation Flight Academy and the Civil Aviation Data Communication Co., Ltd. and the Third Research Institute of Jiuzhou Electric Group Co., Ltd. Testing and evaluation. In order to test whether the ADS-B system can meet the aviation requirements, after the approval of the relevant departments, the experiment was carried out in December 2009, and the stability of the ADS-B system rate reduction accuracy calibration, position accuracy, azimuth accuracy and so on were tested. After testing, the various data information collected by the "domestic ADS-B system" meets the design requirements and meets the development needs of the aviation industry. At this stage, the Central South Regional Administration has begun to promote the use of ADS-B technology. Compared with Australia, China's work on ADS-B technology application, ground system construction, flight personnel training, and air traffic control personnel training is still not perfect. With the continuous development of society and science and technology, China has also begun to study advanced surveillance technology in order to improve the level of air traffic management and promote the healthy development of the aviation industry. 


\section{Problems with ADS-B technology application}

\subsection{Technical system}

In the ADS-C technical system, the transmission of the ADS track report needs to meet certain conditions. Compared with the ADS-C technology, the ADS-B technology has different differences in the contract and communication protocol management and control methods of the two monitoring technologies. On the contrary, the difference between the target transmission position and the aircraft navigation information content of the two monitoring technologies is small. The study found that staff members can set their reporting information elements, reporting periods, etc. before the onboard ADS reporting system runs. After receiving the message sent by the ground control department, the airborne device can send the downlink message to the ground control department, so that the ADS system can realize the information bidirectional data link transmission target. In short, in order to ensure that the ground control department or the airline dispatched and other ground users can timely grasp the aircraft position, air pressure, flight speed and other information, and timely control and control its route and navigation speed, it is necessary to set the aircraft and route feedback content and interval in detail. At the same time, in order to ensure that the ground users can obtain relevant data information in time, the ground users need to cooperate with relevant operators to clarify their information transmission services. The study found that as the ground users continue to increase the channel information, data information elements, and the number of reports, the user's payment of information service fees will gradually increase. In the context of this technical system, although advanced monitoring technology can meet the needs of terrestrial users, due to the high operating cost of ADS monitoring technology, there are fewer enterprises and departments that use this technology.

\subsection{Technical compatibility}

First, the difference in the two-way communication system. ADS-B technology adopts the broadcast two-way communication method. China chooses the AEEC618/AEEC622 protocol to design the track tracking and control data communication data link. The data update efficiency of this communication method will be affected and constrained by the response protocol, and its data. The information is not in real time (cannot meet the requirements of high-density flight management work), and this communication method is not fully compatible with ADS-B technology;

Second, the data chain capacity is different. In order to ensure that ADS-B technology can meet the requirements of air traffic control and airline navigation management, it is necessary to ensure that its data link technology can meet the requirements of monitoring high-density flight work, so the data length and communication efficiency must be strictly controlled. The broadcast data link $1090 \mathrm{MHzS}$ mode, which is more respected by the International Aviation Organization and compatible with ADS-B technology, can fully expand its teletext data link. At this stage, the downlink data length and maximum data rate of the ground-to-air data link adopted in China are significantly lower than the broadcast data link, so the ground-to-air data link is not compatible with ADS-B technology;

Third, the difference in transmission technology. ADS-B broadcast messages have high transmission efficiency, and their downlink data needs to be transmitted to ground users such as air traffic control and airline operation dispatch after being transmitted to the ground users. The monitoring system currently used in China requires the use of satellites for collecting downlink data information, and these data information can be transmitted to the user terminal after being processed reasonably. This information transmission method has low efficiency and the data 
transmission has a delay, which cannot meet the requirements of high-density flight monitoring work.

In order to ensure that the existing monitoring system is fully compatible with ADS-B technology, the data link system that can be selected by the staff is: a new air-to-air, ground-to-air data link system. The data link has an important impact on the promotion and development of ADSB technology. At this stage, countries and organizations around the world have developed a variety of data link systems based on their own needs and intentions. To ensure that airborne equipment and ground equipment can fully play their functions, China needs to rationally select data links that meet the development needs of China's aviation industry system. The study found that all countries in the world choose different data links according to their actual needs and technology level, but they are mainly divided into the following categories: First, the VHF data link model is widely used in Europe. Its main technology is the SOTDMA protocol; secondly, the United States uses a wider range of universal radio data links. It is mainly used in general aviation aircraft; however, there is also an important issue in this data link: interference problems between the data link and the ground equipment; finally, the $1090 \mathrm{MHzS}$ mode extended teletext data link promoted by ICAO. It is a twoway data communication system, and its disadvantage is that the spectrum is used too much.

ICAO has been working hard to develop data link standards that can be used by countries to develop data link systems that can be used globally. If aircraft from all over the world use the same data link system, pilots can fully understand the aviation equipment around the aircraft they are driving to ensure the safety of the aircraft.

\section{Analysis of the development prospects of ADS-B system}

At this stage, China's air transport industry is developing at a faster rate. The number of aircraft, aircraft types and flight areas are also increasing, and the air traffic control workload is also increasing. At present, China has not mastered the core technology of the ADS-B system, and needs to introduce foreign advanced technology, which will increase the operating cost of the ADS-B system. Therefore, China needs to strengthen research on ADS-B technology. In addition, China needs to consider the ADS-B technology cost and its economic benefits in detail, and optimize the existing air traffic control system. In the future, China will inevitably need to popularize ADS-B technology, improve the level of air traffic management, and ensure people's travel safety. China's aviation management departments need to give full play to their role, consider various factors in detail, and scientifically plan ADS-B technology promotion systems and measures.

\section{Conclusion}

In short, the application of ADS-B technology to the aviation industry can improve the level of air traffic management and enhance the effectiveness of aviation management. Using ADS-B technology can help pilots understand the location and related information of the aircraft, and can grasp the speed and position of nearby aircraft, and control the distance between other aircraft, which is very important to improve the flight safety of the aircraft.

\section{References}

[1] Fan Zhao. Discussion on the Application of ADS-B in Air Traffic Control [J]. China New Communications, 2016, 18(7): 40-40.

[2] Wang Yunwei. Discussion on the Advantages of ADS-B Technology and Its Prospects [J]. Industrial Technology Innovation, 2017(02): 150-152.

[3] Zhang Yongwang. Research on Architecture of Aviation Surveillance System Based on Beidou RDSS and ADS-B[J]. Computer Knowledge and Technology, 2017(26): 169-170. 
[4] Huang Danjun. Design and Application of ADS-B Technology in INDRA Air Traffic Control Automation System [J]. China High-tech Enterprise, 2017(1): 41-43.

[5] Zhou Zhi. A Brief Introduction to the Principle of ADS-B Technology and Its Development in Civil Aviation [J]. Communication World, 2016(24): 28-29.

[6] Yuan Jiajun. Discussion on the Application of ADS-B in Air Traffic Control [J]. China Market, 2016(36):168-168. 